\title{
New Reactor Neutrino Experiments besides Double-CHOOZ
}

\author{
M. Goodman ${ }^{\mathrm{a}}$ \\ ${ }^{a}$ High Energy Physics Division \\ HEP 362
}

Argonne Illinois 60439

Several new reactor neutrino experiments are being considered to measure the parameter $\theta_{13}$. The current plans for Angra, Braidwood, Daya Bay, KASKA and KR2DET are reviewed. A case is made that, together with Double-CHOOZ, a future world program should include at least three such experiments.

\section{Introduction and Remarks about $\theta_{13}$}

The mixing angle $\theta_{13}$ is the last mixing angle to be measured. A non-zero value of $\theta_{13}$ is key to the possibility of measuring $\mathrm{CP}$ violation in $\mathrm{fu}-$ ture long-baseline accelerator experiments. The best limit on $\theta_{13}$ comes from the reactor experiment CHOOZ. [1] It has recently been recognized that the best possibility for rapidly measuring a non-zero value of the parameter $\theta_{13}$ comes from a new reactor experiment using two or more identical detectors and improved control of systematic errors.

During 2003, a group of neutrino physicists assembled for a series of workshops to explore the capabilities of a new nuclear reactor experiment. Together, they wrote a white paper called "A New Nuclear Reactor $\nu$ Experiment to Measure $\theta_{13}$ "2]. Several issues were addressed in this document:

$\odot$ The optimal baseline distances, luminosity scaling and the impact of systematics

$\odot$ Previous reactor Experiments

$\odot$ Detector Design

$\odot$ Calibration requirements and procedures

$\odot$ Detector overburden and backgrounds

$\odot$ Systematic error budget

$\odot$ Possible sites

$\odot$ Other physics that could be addressed in a new experiment
$\odot$ Tunneling issues
$\odot$ Safety
$\odot$ Outreach and Education

The document was written with a siteindependent description of such a new experiment, but seven appendices were included about particular initiatives. In a companion presentation at this meeting, the Double-CHOOZ project was described. 3 That project may be the first one underway, but due to limitations of its size and distance, more sensitive and larger but later projects are also under active development. This paper will describe the plans for some of these "other" experiments.

The possibility of a new reactor experiment to measure $\theta_{13}$ was a key concern of the DNP/DPB/DAP/DPB Joint Study on the Future of Neutrino Physics, which recently issued the report, "The Neutrino Matrix" 4. One of the two high priority recommendations was for "a comprehensive U.S. program to complete our understanding of neutrino mixing, to determine the character of the neutrino mass spectrum and to search for CP violation among neutrinos," including "an expeditiously deployed multi-detector reactor experiment with sensitivity to $\bar{\nu}_{e}$ disappearance down to $\sin ^{2} 2 \theta_{13}=0.01$, an order of magnitude below present limits." The choice of 0.01 reflects the desire to provide unambiguous information about $\theta_{13}$ that would be useful to foreseeable future long-baseline experiments that could measure $\mathrm{CP}$ violation and matter effects. As shown 
in Figure 1 it also reflects the approximate foreseeable sensitivity for the next generation or two of reactor experiments.

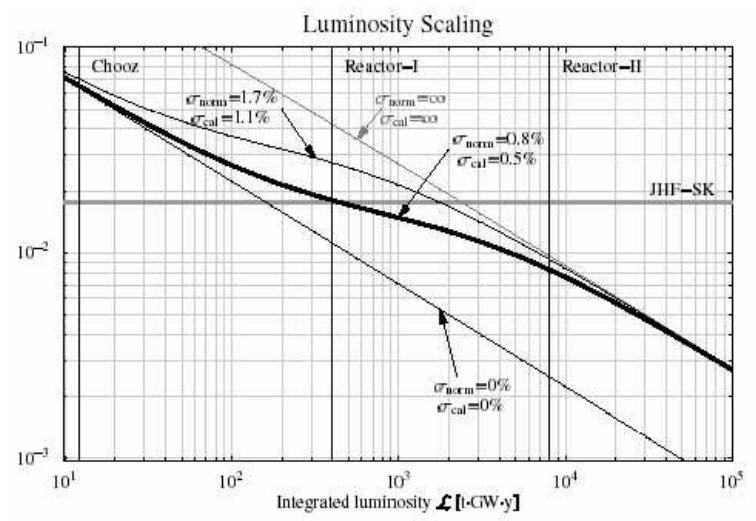

Figure 1. A plot of the $\sin ^{2}\left(2 \theta_{13}\right)$ sensitivity (vertical) versus reactor luminosity (horizontal) for particular detector distances, systematic error assumptions and $\Delta m^{2}$ from Reference [7].

\section{Sites}

\subsection{KR2DET}

The first idea for a new nuclear reactor experiment was the KR2DET project using a reactor at Krasnoyarsk in Russia which utilizes a rapid fuel cycle. [5] Use of a rapid fuel cycle would reduce the systematic error on the flux of neutrinos due to burn up. The most attractive feature of this experiment is that the entire site is located at a depth of 600 meters of water equivalent (mwe). They proposed two detectors, each a $4.7 \mathrm{~m}$ diameter liquid scintillator target, enclosed in a transparent spherical balloon. Each detector would use 8008 -inch photomultipliers, mounted on a stainless steel screen which separates a veto from the inner detector.

The existence of the underground lab together with the reactor is a strong advantage. But this particular reactor will be closed by treaty before the year 2012, because it is of the type that creates weapons grade material; indeed that is what it was built for. It is currently used to provide power for the city of Krasnoyarsk, and will be replaced with an energy plant which cannot be used to create Plutonium. It might have been possible to complete a $300 \mathrm{GW}$-ton-year experiment before the reactor turned off. However a planned visit by western physicists in 2003 was canceled by local security officials, and development of this experiment effectively ended at that time.

\subsection{Diablo Canyon}

The idea for using the Diablo Canyon reactor has been developed by the Berkeley group and others. 6] They did a geological evaluation and tunnel cost estimate for two detectors near this pair of cores with a total $6.1 \mathrm{GW}$-thermal power. The excavation of a horizontal tunnel in the coastal mountains can provide overburden up to 800 mwe with tunnel distances up to $3 \mathrm{~km}$.

Originally the power company, PG\&E, cooperated in the development of the proposal. Construction of an on-site waste disposal facility provided a window of time during which there would be civil construction on site anyway, and the addition of a tunnel would not create additional environmental concerns. However, for reasons that are not clear, PG\&E decided that this project would not be in its interest, and they have discouraged further development of this proposal.

\subsection{KASKA}

The largest concentration of nuclear power plants in the world, and hence the most powerful neutrino source, is at Kashiwazaki-Kariwa, with a total of $24.3 \mathrm{GW}$-thermal. Seven reactors are located along the west coast of Japan. The reactors are located in one cluster of four and one cluster of three. A favorable geometry exists which consists of two near detectors and one far detector, located on the site boundary. The ground is soft, so rather than constructing a cavern underground, a detector would be placed in a shafthole, drilled with the techniques used for making bridges. The experiment, called KASKA, plans to use four volumes of liquid:

1. An inner Neutrino target consisting of 
Gadolinium loaded liquid scintillator.

2. A gamma-catcher consisting of scintillator without Gd.

3. A buffer region consisting of mineral oil without scintillator.

4. An optical barrier before a cosmic ray veto region with weak scintillator.

KASKA recently received some $R \& D$ funding to drill a test hole and make some preliminary cosmic ray studies. They are also developing a prototype detector.

\subsection{Braidwood}

The Braidwood project is an active collaboration of Chicago, Oxford, Kansas State, Columbia, ANL, FNAL, BNL, Pittsburgh, MIT, Michigan and Texas. The Braidwood reactor is a two unit facility located about 50 miles southwest of Chicago. An R\&D proposal for one year has been submitted to the NSF and the DOE and a full proposal is expected in about a year. The baseline design consists of two near and two far detectors with a $3.5 \mathrm{~m}$ outer radius. The distances will be along the center line of the two reactor cores, and approximately $200 \mathrm{~m}$ and $1500 \mathrm{~m}$ from the cores. The planned depth is $180 \mathrm{~m}$ corresponding to 450 mwe. The design currently is for a two zone detector, using $0.1 \% \mathrm{Gd}$ loaded scintillator in the inner volume and mineral oil in the outer volume. The radius of the acrylic sphere will be $2.6 \mathrm{~m}$. There will be approximately $25 \%$ phototube coverage, using 1000 PMTs per detector. The detectors will be movable. Baseline sensitivities will not use the reduced systematic from moving, but this will be used as a cross-check. A veto system of approximately $1 \mathrm{~m}$ of passive shielding surrounded by active veto counters on the top and sides will be used. There will also be a layer of active veto counters below the detectors.

\subsection{Daya Bay}

The Daya Bay complex of reactors is located in China outside Hong Kong. There are two reactors at Daya Bay, two more at Ling Ao, and plans for another two nearby at Ling Dong. Nearby are large hills. Two near detectors would be built, one near Daya Bay and the other between Ling Ao and Ling Dong. A far detector could be located deep in the hills under a considerable overburden of 1200 mwe. When Ling Dong is complete, this will be the second most powerful reactor complex in the world.

\subsection{Angra dos Rois}

Angra dos Reis is located about $150 \mathrm{~km}$ south of Rio de Janeiro in Brazil. The nuclear facility contains two operational reactors. The Angra-I reactor is an older low power reactor $(1.5 \mathrm{GW}$-th). Angra-II is $4.1 \mathrm{GW}$-th. The reactors are located on the coast and the reactor company controls a strip of land that stretches inland about $1-1.5 \mathrm{~km}$ and is approximately $4 \mathrm{~km}$ along the coast. Much of the terrain is mountainous granite with multiple peaks up to $600 \mathrm{~m}$ high. The focus of the design work is now oriented toward large detectors, larger than 100 ton each, in order to take advantage of the spectral shape test. This means that energy calibration will be crucial. The next workshop of the International Working Group is tentatively scheduled for 23-25 February 2005 near Angra, Brazil.

\subsection{Common concerns}

The experiences at Diablo Canyon and Krasnoyarsk emphasizes that there has been a history of less than stellar cooperation between reactor power companies/authorities and reactor neutrino experiments. The cases are all different, but include San Onofre, Palo Verde, Krasnoyarsk, and Diablo Canyon. An appreciation of the needs and concerns of the reactor authorities is crucial in developing a new larger program. These issues include security, safety and economics. So far, the other reactor operators: Electricite de France, Tokyo Electric Power, Exelon, and the governments of China and Brazil have been more willing to cooperate with the physicists investigating new experiments. Careful and continued consideration to these concerns is crucial for a successful ambitious future program of neutrino reactor experiments.

\subsection{Comments}

A summary of proposed experiment parameters is given in Table 1] As funding agencies 
Table 1

\begin{tabular}{|l|l|c|c|c|}
\hline Proposal & Power & Baseline & Detector & Overburden \\
\hline & $(\mathrm{GW})$ & Near/Far $(\mathrm{m})$ & Near/Far $(\mathrm{t})$ & Near/far $(\mathrm{mwe})$ \\
\hline Angra dos Reis (Brazil) & 4.1 & $300 / 1300$ & $25 / 25$ & $60 / 600$ \\
\hline Braidwood (US) & 6.5 & $200 / 1500$ & $25 / 50$ & $250 / 250$ \\
\hline Double-CHOOZ (France) & 8.4 & $150 / 1050$ & $10 / 10$ & $50 / 300$ \\
\hline Daya Bay (China) & 11.6 & $300 / 1500$ & $25 / 50$ & $200 / 1000$ \\
\hline Diablo Canyon (US) & 6.4 & $400 / 1800$ & $25 / 50$ & $100 / 700$ \\
\hline KASKA (Japan) & 24.3 & $300 / 1300$ & $8.5 / 8.5$ & $140 / 600$ \\
\hline KR2DET (Russia) & 3.2 & $115 / 1000$ & $46 / 46$ & $600 / 600$ \\
\hline \hline
\end{tabular}

Detector parameters for proposed experiments. Note that except for the reactor powers, and the DoubleChooz far detector distance and overburden, and the KR2DET overburden, all parameters are subject to design and optimization.

try to evaluate the best strategy, it is important to keep in mind that except for power, most entries there are subject to design and optimization. The optimum strategy for a future program of reactor neutrino experiments includes consideration of detector sizes and systematic errors, but also costs and institutional and national considerations, not fully considered in this presentation.

A glance at Figure 11 indicates some of the challenges. The thick curve represents the sensitivity/luminosity relation using a reasonable guess for the ultimate systematic errors of a new experiment. [7] Note that the luminosity (in GWt-years) for a sensitivity of 0.01 is 70 times larger than the luminosity for a sensitivity of 0.03 , which is the goal for Double-CHOOZ. I think a first experiment such as Double-CHOOZ is a prerequisite for the more sensitive experiment, whether it meets its goal by increased luminosity or by decreased systematic errors. But I also think the story won't stop there. Just as experiments to increase knowledge of the elements of the CKM matrix are continuing, better knowledge about $\theta_{13}$ will be a continued goal of the particle physics community for some time to come, whether or not a non-zero value is found soon. I foresee at least three new rounds of neutrino reactor experiments, each building on the lessons of the previous experiments.

\section{REFERENCES}

1. M. Apollonio et al., Eur. Phys. J. C27 (2003) 331.

2. K. Anderson et al., "White Paper Report on a New Nuclear Reactor $\nu$ Experiment to Measure $\theta_{13} "$, January 2004, hep-ex/0402041.

3. G. Mention, these proceedings.

4. "The Neutrino Matrix", S. Freedman and B. Kayser chairmen of the organizing committee, physics/0411216

5. V. Martemyanov et al., hep-ex/0211070 2002.

6. Appendix D of Reference 2].

7. P. Huber et al., Nucl.Phys. B665 (2003) 487. 